\title{
Severe generalised hypersensitivity reaction to topical neomycin after cataract surgery: a case report Imran A Ansari* and Ernest Onyema
}

\author{
Address: Department of Ophthalmology, Princess Alexandra Hospital, Harlow, Essex, UK \\ Email: Imran A Ansari* - dociansari@hotmail.com; Ernest Onyema - eyemd121@gmail.com \\ * Corresponding author
}

Published: 23 February 2008

Journal of Medical Case Reports 2008, 2:57 doi:10.1 186/1752-1947-2-57
Received: 9 July 2007

Accepted: 23 February 2008

This article is available from: http://www.jmedicalcasereports.com/content/2/1/57

(c) 2008 Ansari and Onyema; licensee BioMed Central Ltd.

This is an Open Access article distributed under the terms of the Creative Commons Attribution License (http://creativecommons.org/licenses/by/2.0), which permits unrestricted use, distribution, and reproduction in any medium, provided the original work is properly cited.

\begin{abstract}
Introduction: Systemic hypersensitivity reactions to topical ophthalmic treatment occur rarely, but when they do they can be severe as highlighted by this case.

Case presentation: A post-operative cataract surgery patient developed a severe and generalised hypersensitivity reaction following topical treatment with Maxitrol (Dexamethasone and Neomycin) eye drops. The patient reported a previous allergic reaction to Neomycin.
\end{abstract}

Conclusion: This case report emphasises the importance of a thorough drug and allergy history when patients are seen at pre-assessment or clerked in for surgery.

\section{Introduction}

Adverse external ocular effects of topical ophthalmic therapy have been estimated to occur in $10 \%$ of all adverse reactions [1].

Drug-related ocular allergies are often the result of type IV hypersensitivity reactions, although type 1 and type 3 hypersensitivity reactions may also be involved [2].

Hypersensitivity to localised ocular therapy may involve a localised contact reaction which may include itching, redness, tearing, mucopurulent discharge, and papillary conjunctivitis as well as corneal involvement [3]. Dermatitis, oedema and chemosis of the eyelids and skin can also occur [4].

Anaphylactoid reactions are rare and can be the result of type 1 (immediate) hypersensitivity reactions [1]. They are not usually associated with systemic anaphylaxis but involve an acute shock syndrome that may be immunologically mediated [5].
Anaphylactoid reactions are characterised by acute itching, conjunctival hyperemia, chemosis and oedema of skin in the form of urticaria and angioedema Neomycin is an antibiotic that is often used in the form of Maxitrol (Neomycin, Polymyxin B and Dexamethasone) after cataract surgery in the prophylaxis of infection.

When used in ophthalmic preparations, it is well known to be a cause of allergic localised contact reactions, but it is rare for it to cause a diffuse cutaneous systemic reaction [6]. One study has estimated the incidence of allergic contact reactions to topical neomycin as 1 to 29/100,000 [7]. We describe a case of severe systemic allergy with a diffuse cutaneous hypersensitivity reaction following topical ophthalmic administration of neomycin in the form of Maxitrol.

\section{Case presentation}

An 80 year old Caucasian male underwent routine phacoemulsification cataract extraction with posterior chamber intraocular lens implant. 
The patient had a history of hypertension and hypercholesterolemia for which he was using Atenolol and Simvastatin. He also had a history of bladder cancer for which he had received chemotherapy and radiotherapy.

Of note, the patient had reported an allergic reaction to Neomycin ear drops 30 years previously. This had left him with a rash that improved a few days later.

The patient was discharged after a straight forward cataract operation on Maxitrol eye drops four times a day.

He was seen the next day for the first post-operative visit, and up until then had used Maxitrol twice to the affected eye.

At that stage the patient's eye was noted to be slightly more injected than usual but no further concerns were raised. Papillae were noted on the palpebral conjunctiva and treatment was continued.

The patient was then reviewed 2 weeks post-operatively and had continued to use Maxitrol up until that time. He was found to have increased facial erythema, swelling and crusting around the lids. (see Fig. 1). He also complained of a severe and generalised rash affecting his legs, arms, back and buttocks which was painful. (Figs. 2 and 3). No other mucus membranes were involved.

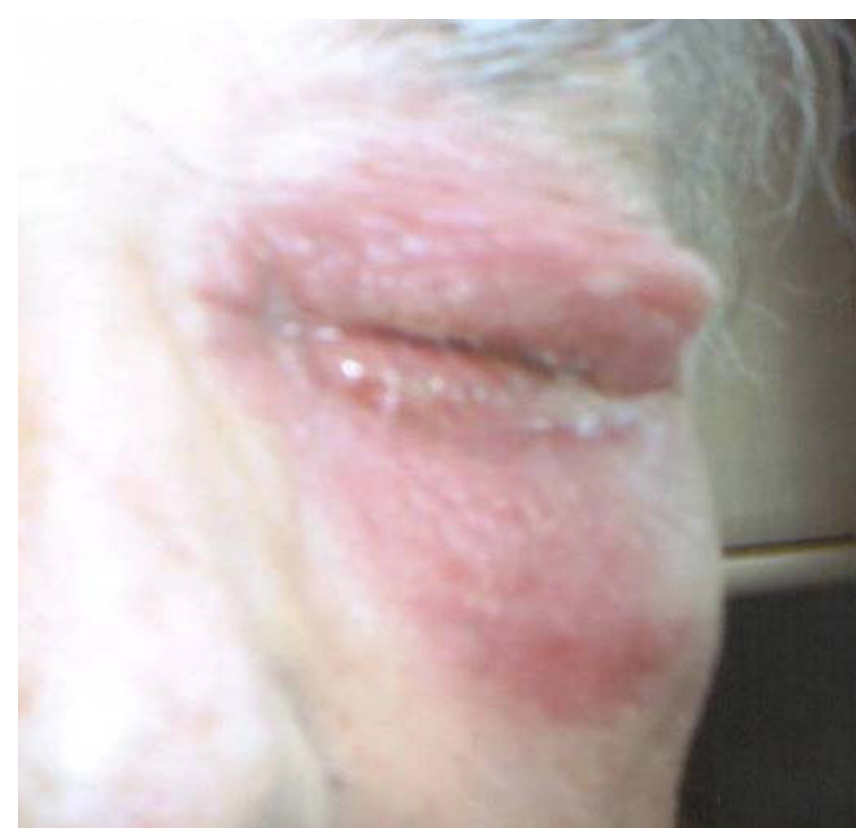

Figure I

Severe pruritic periorbital rash and swelling around left eye.

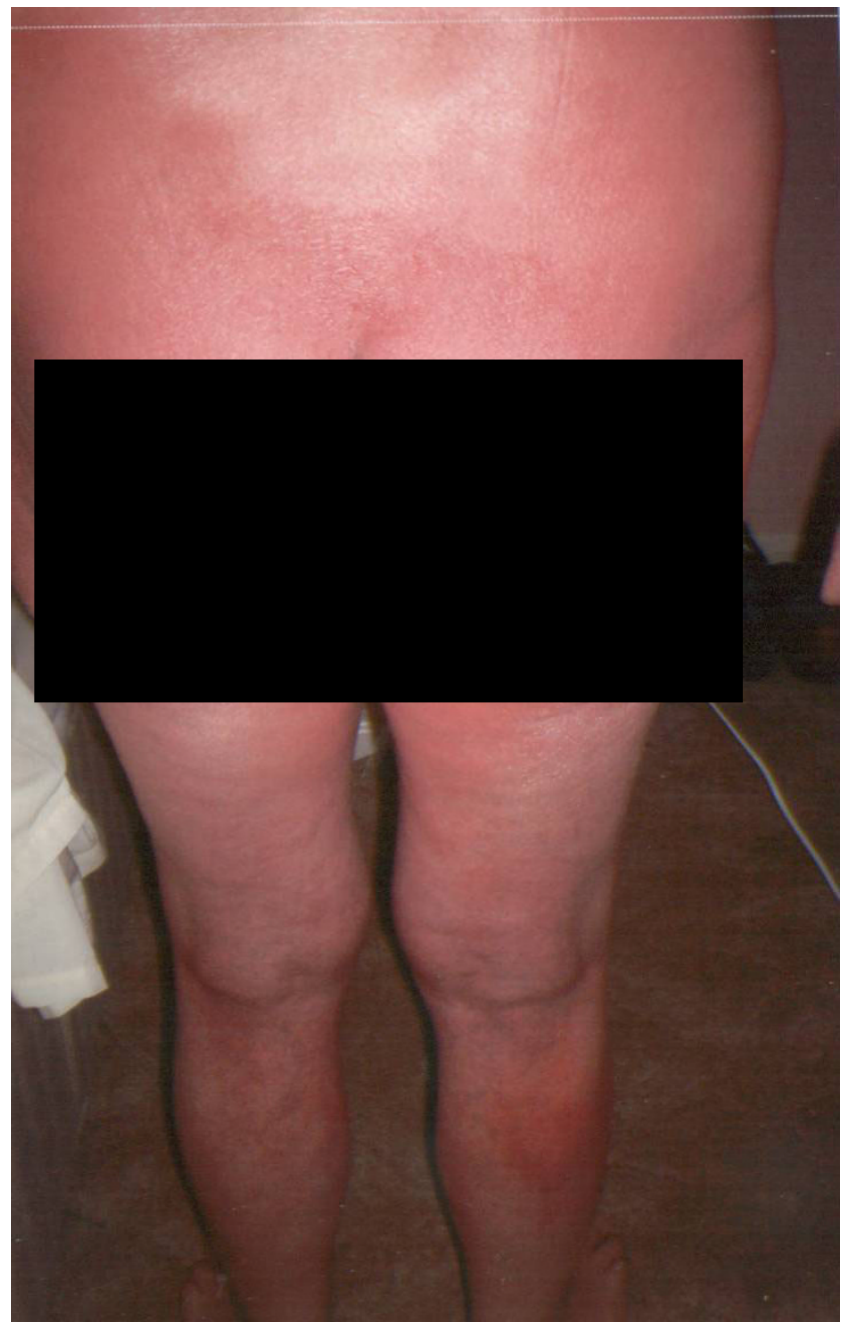

Figure 2

Generalised hypersensitivity reaction involving the trunk and lower limbs.

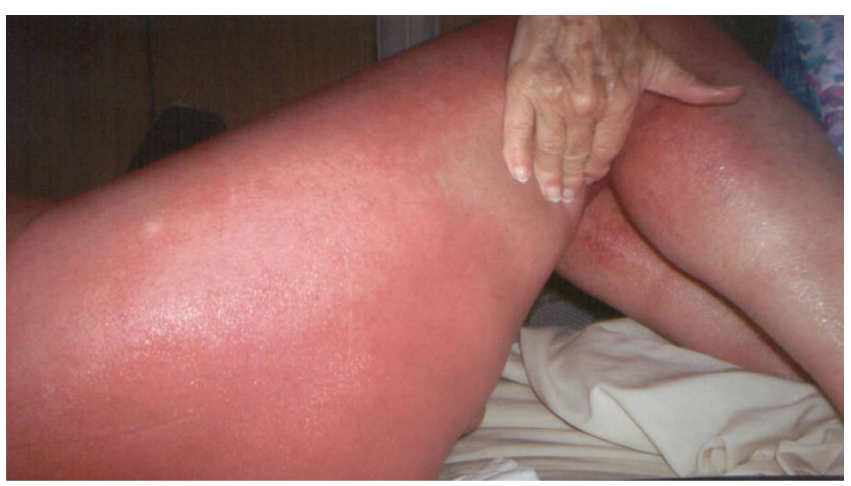

Figure 3

Maculo-papular rash - Involvement of the lower limb. 
Treatment with systemic Prednisone $20 \mathrm{mg}$ once a day and Betnovate eye ointment relieved his symptoms remarkably, and 3 weeks later all signs of inflammation were gone.

\section{Conclusion}

Systemic hypersensitivity reactions to topical ophthalmic treatment occur rarely. but when they do they can be severe as highlighted by this case.

Although the patient in this report had a substantial improvement in best-corrected visual acuity from counting fingers pre-operatively to $6 / 9$ post-operatively, he endured a long and painful recovery which otherwise would have been quick and straight forward. An alternative post-operative regime could have been Pred Forte and Chloramphenicol eye drops four times a day.

This case report emphasises the importance of a thorough drug and allergy history when patients are seen at preassessment or clerked in for surgery. Even when drugs are given topically and locally, the possibility of a severe systemic reaction should always be borne in mind even though these are rare. As physicians it is also important for us to educate our patients about the signs and symptoms of such an allergic reaction so the patient will return to us sooner for treatment.

\section{Competing interests}

The author(s) declare that they have no competing interests.

\section{Authors' contributions}

IAA was the main author and is the corresponding author. EO is the co-author. All authors read and approved the final manuscript.

\section{Consent}

Written informed consent was obtained from the patient for publication of this Case report and accompanying images. A copy of the written consent is available for review by the Editor-in-Chief of this journal.

\section{Acknowledgements}

No funding was necessary for this article.

\section{References}

I. Wilson FM II: Adverse external ocular effects of topical ophthalmic therapy: an epidemiologic, laboratory, and clinical study. Trans Am Ophthalmol Soc 1983, 81:854-965.

2. Wilson, Fred M II: Allergy to Topical Medications. International Ophthalmology Clinics. Recent Trends in Ocular Allergy 2003, 43(I):73-8I. Winter

3. Wilson FM II: Adverse external ocular effects of topical ophthalmic medications. Surv Ophthalmol 1979, 24:57-88.

4. Stern GA, Knapp A: Iatrogenic peripheral corneal disease. Int Ophthalmol Clin 1986, 26:77-89.

5. Wise DJ, Carter GR: Immunology. A comprehensive review. Ames, lowa: lowa State University Press; 2002.
6. Baldinger J, Weiter J]: Diffuse cutaneous hypersensitivity reaction after dexamethasone/polymyxin B/neomycin combination eye drops. Source Annals of Ophthalmology 1986, 18(3):95-6.

7. de Pádua CA, Uter W, Schnuch A: Contact allergy to topical drugs: prevalence in a clinical setting and estimation of frequency at the population level. Pharmacoepidemiol Drug Saf 2007, 16(4):377-84.
Publish with BioMed Central and every scientist can read your work free of charge

"BioMed Central will be the most significant development for disseminating the results of biomedical research in our lifetime. " Sir Paul Nurse, Cancer Research UK

Your research papers will be:

- available free of charge to the entire biomedical community

- peer reviewed and published immediately upon acceptance

- cited in PubMed and archived on PubMed Central

- yours - you keep the copyright

Submit your manuscript here:

http://www.biomedcentral.com/info/publishing_adv.asp
BioMedcentral 